\title{
Rinosinusitis fúngica invasiva aguda: Actualización en pacientes adultos y pediátricos
}

\author{
Acute invasive fungal rhinosinusitis: Update in adults and pediatric patients
}

\author{
Maximiliano Penna $\mathbf{R}^{1}$, Luis Barahona $\mathbf{A}^{2}$, Paula Ruz $\mathbf{M}^{1,3}$, Constanza Valdés $\mathbf{P}^{1,4}$.
}

\begin{abstract}
RESUMEN
La rinosinusitis fúngica invasiva aguda (RSFIA) es una enfermedad poco frecuente caracterizada por una infiltración fúngica de la submucosa y vasos sanguíneos de las cavidades nasal y paranasal. Afecta a pacientes con grados variables de inmunosupresión, destacando entre estas patologías subyacentes la diabetes mellitus y las neoplasias malignas hematológicas. Presenta una alta tasa de mortalidad, pudiendo reducirse significativamente si el diagnóstico y el tratamiento se realizan precozmente. Este artículo tiene por objetivo presentar una revisión actualizada de la literatura respecto a la presentación clínica, microbiología, factores de riesgos, métodos diagnósticos, tratamiento y pronóstico de la RSFIA, tanto en adultos como en niños.
\end{abstract}

Palabras clave: Sinusitis fúngica invasiva, agentes antifúngicos, senos paranasales, huésped inmunocomprometido, rinitis, debridamiento.

\section{ABSTRACT}

Acute invasive fungal rhinosinusitis (AIFS) is a rare disease characterized by fungal infiltration of the submucosa and blood vessels of the nasal y paranasal cavities. It affects almost exclusively patients with different degrees of immunosuppression, with underlying pathologies such as diabetes mellitus and hematological malignancies. AIFS has a high mortality rate, but it can be significantly reduced if the diagnosis and treatment are carried out early in the course of disease. This article aims to present an updated literature review regarding clinical presentation, microbiology, risk factors, diagnostic methods, treatment and prognosis of AlFS, both in adults and children.

Key words: Acute invasive fungal rhinosinusitis, antifungal agents, paranasal sinuses, immunocompromised host, rhinitis, debridement.

\footnotetext{
Servicio de Otorrinolaringología Hospital del Salvador, Universidad de Chile, Santiago, Chile.

2 Escuela de Medicina, Universidad de Chile, Santiago, Chile.

3 Servicio de Otorrinolaringología, Clínica Vespucio, Santiago, Chile.

4 Servicio de Otorrinolaringología, Clínica Las Condes, Santiago, Chile.
}

Los autores declaran no tener conflictos de interés. 


\section{INTRODUCCIÓN}

La rinosinusitis fúngica puede existir en formas no invasivas e invasivas. Las formas no invasivas incluyen la bola fúngica, saprofítica, y sinusitis fúngica alérgica ${ }^{1}$. La rinosinusitis fúngica invasiva se puede clasificar en tres categorías: crónica invasiva, granulomatosa, y rinosinusitis fúngica invasiva aguda (RSFIA) ${ }^{2}$. La clasificación de la rinosinusitis fúngica se representa en la Figura 1.

La RSFIA es una entidad rara que se desarrolla en un plazo menor a 4 semanas, caracterizada por infiltración micótica progresiva de la submucosa de las cavidades nasal y paranasal, que puede invadir a los vasos sanguíneos y causar necrosis tisular. En ausencia de tratamiento es rápidamente fatal en $50 \%-80 \%$ de los casos ${ }^{1}$. La enfermedad ocurre principalmente en pacientes inmunocomprometidos. Las asociaciones más comunes incluyen diabetes no controlada, neoplasias malignas hematológicas, uso prolongado de glucocorticoides e infección por el virus de la inmunodeficiencia humana $(\mathrm{VIH})^{3}$, llegando a tener una incidencia en esta población de riesgo de $1 \%$ a $4,6 \%{ }^{4}$. El cornete medio es una de las zonas más frecuentemente comprometidas, siendo reportada su invasión fúngica al menos 2 veces más que en otros sitios comúnmente afectados, como el seno maxilar y etmoidal ${ }^{5}$.

Los pacientes que presentan sinusitis fúngicas invasivas suelen presentar síntomas inespecíicos que se pueden observar en otras patologías rinosinusales menos agresivas, por lo que, si no se tiene un umbral bajo de sospecha en pacientes de riesgo, se podría retrasar el diagnóstico y comprometer con ello el pronóstico $0^{6}$. La RSFIA sigue siendo uno de los procesos de enfermedad que tienen más impacto en la población inmunocomprometida, debido a su alta agresividad, morbilidad y mortalidad, por lo que el diagnóstico precoz cumple un rol primordial. El papel del otorrinolaringólogo es clave tanto en el diagnóstico como en el tratamiento de esta patología.

\section{Microbiología}

Los principales microorganismos aislados en la RSFIA pertenecen a las divisiones Zygomycota (Mucor, Rhizopus, rhizomucor, Absidia y otros hongos mucorales) y Ascomycota (especies de Aspergillus), agentes encontrados saprofíticamente en sustancias descompuestas, suelo y frutas, además de la vía aerodigestiva superior y heces de individuos sanos ${ }^{7}$. No se ha observado una diferencia significativa en cuanto a la morbimortalidad, en aquellos pacientes infectados por Aspergillus comparados con los infectados por hongos mucorales ${ }^{1,8}$.

\section{Factores de riesgo}

Dado que la enfermedad se desarrolla principalmente en pacientes inmunodeprimidos, las

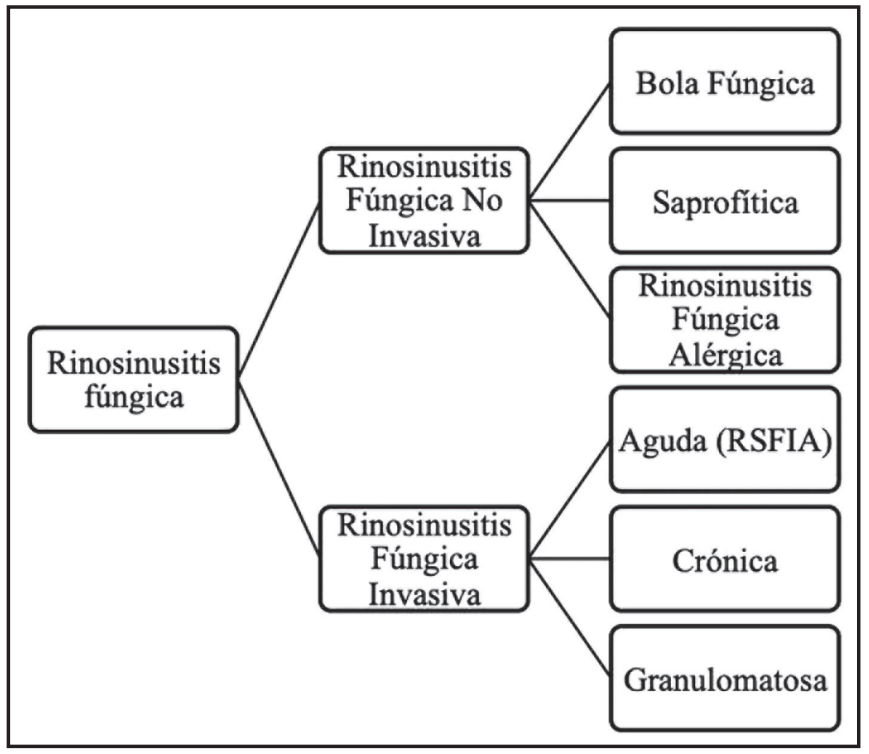

Figura 1. Clasificación de la rinosinusitis fúngica. 
distintas publicaciones muestran asociación de la RSFIA con diversas comorbilidades que favorecen este estado. En una revisión sistemática, Turner y cols reportaron que la condición predisponente más frecuente fue la diabetes mellitus, con 47,8\% de los pacientes, presentándose la mitad de ellos con cetoacidosis diabética ${ }^{3}$. Otros factores predisponentes fueron las neoplasias malignas hematológicas (39\%), el uso de corticoesteroides $(27,6 \%)$, la insuficiencia renal o hepática $(6,6 \%)$, el trasplante de órganos sólidos (6,3\%), el VIH/SIDA $(2,3 \%)$ y las enfermedades autoinmunes $(1,2 \%)^{3}$. Todas las condiciones previamente descritas suponen grados variables de inmunosupresión. Se ha visto que la neutropenia severa, definida como un recuento absoluto de neutrófilos (RAN) menor a $500 \mathrm{cél} / \mathrm{mL}$, predispone a enfermedades oportunistas como RSFIA, y, por lo tanto, la recuperación de este estado inmunosupresor es un pilar fundamental en el tratamiento $0^{9,10}$.

\section{Cuadro clínico}

El cuadro clínico inicial tiende a ser inespecífico, similar a otras patologías más inocuas como por ejemplo la rinosinusitis aguda viral o bacteriana, dada la presencia de síntomas como congestión nasal, rinorrea, cefalea y algia facial. Por lo tanto, el reconocimiento de estos síntomas en población de riesgo requiere un alto índice de sospecha ${ }^{10}$. En una revisión sistemática se describe el aumento de volumen facial como principal manifestación clínica $(64,5 \%)$, seguido de fiebre $(62,9 \%)$, congestión nasal $(52,2 \%)$, oftalmoplejía (50,9\%), proptosis $(48,9 \%)$, disminución de agudeza visual $(48,9 \%)$, descarga nasal $(48,1 \%)$ y algia facial $(46,8 \%) 3$. Estos signos y síntomas evidenciarían una etapa más avanzada de la enfermedad, describiéndose además con la progresión la disfunción del nervio facial, dolor dental, induración palatina, alteración de conciencia y convulsiones ${ }^{10}$. Se ha descrito también que la fiebre de origen desconocido podría ser la manifestación única de hasta el $52 \%$ de los pacientes ${ }^{5}$.

El examen endoscópico nasal es esencial en la evaluación de pacientes susceptibles a una RSFIA, sin embargo, a menudo resulta normal previo al desarrollo de una enfermedad invasiva grave ${ }^{5}$. Los hallazgos inicialmente pueden ser muy suti- les, sin embargo, los más descritos son cambios variables de coloración, granulación y ulceración de la mucosa. La coloración blanca que no sangra sugiere isquemia tisular secundaria a invasión angiocéntrica, mientras que la decoloración negra es un signo tardío sugerente de necrosis ${ }^{11}$. Otros hallazgos comunes son la presencia de costras necróticas nasales y secreción mucosa hialina 0 espesa, generalmente provenientes del meato y cornete medio, seguido del cornete inferior y el septum nasal ${ }^{8}$.

\section{Imagenología}

El hallazgo más precoz en la RSFIA es el engrosamiento mucoso unilateral de la cavidad nasal $^{12}$, sin embargo, tiene una baja especificidad ${ }^{13}$. Tradicionalmente se consideraba a la dehiscencia 0 erosión ósea como hallazgo central sugerente de RSFIA debido a su especificidad cercana al $100 \%$, teniendo por ello la tomografía computarizada (TC) un rol esencial ${ }^{13}$. Sin embargo, ha demostrado ser un hallazgo tardío y tener una sensibilidad en torno al $35 \%$, pudiendo incluso existir invasión de la grasa periantral e intraorbitaria con paredes óseas intactas ${ }^{12,13}$. Este hecho es consistente con la capacidad de los hongos de diseminarse no solo mediante extensión directa, sino además a través de canales vasculares y nervios ${ }^{13}$. En un estudio retrospectivo de pacientes con RSFIA por Mucor, se constató que de 6 pacientes que requirieron debridamiento óseo por los hallazgos intraoperatorios, solo 1 tenía erosión ósea en la TC preoperatoria ${ }^{14}$.

Debido a lo anterior, la resonancia magnética (RM) ha adquirido mayor importancia, dado que la anormalidad de los tejidos blandos extrasinonasales podría ocurrir más precozmente en el curso de la RSFIA y traducirse así en un diagnóstico más precoz. En un estudio retrospectivo reciente se constató que, a pesar de tener una especificidad similar, la RM es más sensible en la detección precoz de RSFIA en comparación a la TC, siendo la invasión perisinusal el parámetro único más sensible y específico ${ }^{15}$. En la RM, el realce leptomeníngeo puede ser interpretado como invasión intracraneal, pudiendo progresar la infección generando cerebritis, abscesos cerebrales y granulomas, representándose estos últimos como 
lesiones hipointensas en T1 y T2 con realce mínimo al contraste con gadolinio ${ }^{16}$.

En un estudio que evaluó las características de la RM en pacientes con RSFIA, se constató que el compromiso extranasosinusal sería frecuente, afectando en $65 \%$ de los casos a la órbita ${ }^{17}$. La ausencia de realce con el contraste ocurriría en casi la mitad de los pacientes ${ }^{17}$, lo que podría ser explicado por la naturaleza angioinvasiva de la infección fúngica ${ }^{12}$. Este patrón de comportamiento al contraste además se asoció significativamente a mayor mortalidad, en comparación con los patrones homogéneo y heterogéne $0^{17}$. En un estudio que evaluó las características tomográficas, se encontró que la presencia de erosión ósea, el engrosamiento de partes blandas, la extensión extrasinusal y el compromiso unilateral tienen un valor predictivo positivo (VPP) de $100 \%$, mientras que los cambios inflamatorios mucoperiósticos de los senos paranasales tienen un valor predictivo negativo (VPN) de $100 \%{ }^{4}$. Con la finalidad de elaborar una herramienta más objetiva en la aproximación diagnóstica de la RSFIA, un estudio retrospectivo reciente elaboró un modelo de 7 variables, considerando 4 hallazgos tomográficos tradicionalmente descritos (compromiso de grasa periantral, fosa pterigopalatina, dehiscencia ósea e invasión orbitaria) y 3 características descritas con menor frecuencia (compromiso de conducto nasolagrimal, saco lagrimal y ulceración septal). Este modelo, creado a partir de la comparación de pacientes con RSFIA respecto a controles de la misma población de alto riesgo, reportó una especificidad y VPP de $100 \%$ ante la presencia de 2 variables positivas ${ }^{13}$.

\section{Diagnóstico}

El examen histopatológico diferido es el gold standard en el diagnóstico de la RSFIA, evidenciándose una mucosa con infarto vascular secundario a trombosis por angioinvasión fúngica, acompañado usualmente de escasas células inflamatorias. Las tinciones de plata y PAS pueden ayudar a evidenciar formas fúngicas en paredes y lúmenes vasculares ${ }^{18}$. Este examen se debería realizar ante cualquier anormalidad presente en la evaluación endoscópica, sin embargo, se ha planteado la toma de biopsias en pacientes con factores de riesgo y sospecha alta incluso con tejidos aparentemente normales ${ }^{19}$. Se ha sugerido la biopsia de cornete medio, considerando que es el lugar más frecuentemente reportado, alcanzando una sensibilidad de $75 \%$ a $86 \%$ y especificidad de $100 \%^{5,8,19}$.

Sin embargo, el diagnóstico mediante las secciones permanentes diferidas contempla un tiempo de espera significativo, lo que puede retrasar el tratamiento adecuado y así aumentar la morbimortalidad por la enfermedad. En este contexto, se han planteado métodos diagnósticos más rápidos como el análisis de muestras de sección congela$\mathrm{da}$, que, aunque no es tan precisa como la biopsia diferida, ha demostrado tener buen rendimiento en el diagnóstico de la RSFIA, reportándose una sensibilidad de $87,5 \%$, especificidad de $100 \%$, VPN de $70 \%$ y VPP de $100 \%{ }^{9}$. El análisis de muestras de sección congelada también puede ser considerada una herramienta efectiva para guiar la decisión intraoperatoria en el debridamiento endoscópico. Sin embargo, el VPN relativamente bajo sugiere que los resultados negativos no necesariamente descartan la presencia de invasión fúngica, por lo que el manejo quirúrgico adicional debe basarse además en la sospecha clínica y los hallazgos endoscópicos intraoperatorios ${ }^{20}$. Un estudio reciente comparó los resultados obtenidos con la sección congelada usando tinciones habituales (hematoxilina y eosina) con respecto a la biopsia deferida, además de utilizar de manera retrospectiva y prospectiva una tinción de PAS para hongos modificada para usar en tejidos congelados (PASF-fs). El uso de esta tinción permitió aumentar la sensibilidad de los análisis de sección congelada desde 85,4\% al 95,2\%, registrándose además una especificidad de $100 \%$. Este resultado si bien es alentador, no resultó ser estadísticamente significativo, lo que podría ser explicado por el pequeño tamaño muestral del estudio ${ }^{19}$.

Se han estudiado además otros exámenes diagnósticos como los niveles plasmáticos de galactomanano y el análisis de expresión nasal de maspina. Se ha reportado que la medición plasmática de galactomanano, polisacárido presente en la pared celular de especies de Aspergillus, tendría un rol más específico en la detección de aspergilosis invasiva, teniendo el valor de corte de 0,48 una sensibilidad de $71,4 \%$ y especificidad de 
$93 \%{ }^{21}$. Por otra parte, mediante análisis de inmunohistoquímica en mucosa nasal, se ha visto que la expresión de maspina está disminuida en rinosinusitis fúngica invasiva (incluyendo casos agudos y crónicos), en comparación a rinosinusitis crónica y fúngica no invasiva. Un puntaje de tinción de 5,7 tendría una sensibilidad de $91,7 \%$ y especificidad de $88,3 \%$, por lo que podría constituirse como un biomarcador rápido y efectivo en el diagnóstico de infecciones fúngicas invasivas 22 .

\section{Tratamiento}

El tratamiento de la RSFIA requiere revertir la condición inmunosupresora predisponente, a lo que se suma el tratamiento habitual, que generalmente comprende el debridamiento quirúrgico y la terapia antifúngica ${ }^{11}$. El tratamiento antifúngico inicial estándar corresponde a la administración intravenosa de anfotericina $B$ en dosis de 0,25-1,0 $\mathrm{mg} / \mathrm{kg} / \mathrm{día}$, hasta un total de 2-4 gramos en un plazo de 6 a 8 semanas. Dado su potencial nefrotóxico, se recomienda el uso de anfotericina liposomal en dosis de $3-5 \mathrm{mg} / \mathrm{kg} / \mathrm{día}$ en caso de constatarse una creatininemia superior a $2,5 \mathrm{mg} / \mathrm{dL} 0$ ante evidencia de progresión de enfermedad con dosis máxima de terapia estándar ${ }^{11}$. Las excepciones al tratamiento inicial con anfotericina $B$ son los casos de RSFIA secundaria a Pseudallescheria boydii 0 Aspergillus, en que se requieren dosis superiores debido a su mayor resistencia, lo que conlleva un riesgo mayor de nefrotoxicidad ${ }^{23}$. En estos casos, el tratamiento de elección es el voriconazol, cuya dosis habitual en adultos es de $6 \mathrm{mg} / \mathrm{kg}$ vía endovenosa 2 veces al día durante el primer día, posteriormente $4 \mathrm{mg} / \mathrm{kg} 2$ veces al día por 7 días, con eventual traslape a terapia oral con $200 \mathrm{mg}$ 2 veces al día. Es importante considerar que este esquema es solo válido para estas excepciones, dada la baja actividad del voriconazol en la división Zygomicota ${ }^{23}$.

Por otra parte, el debridamiento quirúrgico permite cumplir múltiples objetivos. En primer lugar, retrasa la progresión de la enfermedad, dando tiempo a la recuperación de neutrófilos en condiciones inmunosupresoras reversibles. Además, reduce la carga fúngica y permite la toma de cultivos y el diagnóstico histológico ${ }^{5}$. Por lo tanto, el debridamiento nasosinusal endoscópico temprano y agresivo, debe extenderse a las estructuras afectadas hasta que se encuentre tejido vascularizado. En cuanto a la técnica a emplear, un estudio retrospectivo que comparó los resultados de las técnicas abierta y endoscópica demostró una supervivencia global cercana al $50 \%$, independiente del método quirúrgico utilizado ${ }^{7}$. Los autores de este estudio sugieren que la vía endoscópica es preferible en casos diagnosticados en etapas tempranas, al ofrecer una opción menos invasiva a pacientes con un estado de salud deteriorado. Por otra parte, la cirugía abierta se recomendaría a pacientes en que se ha objetivado una extensión intraorbitaria, palatina $y / 0$ intracerebral 7 .

En caso de sospechar enfermedad residual, se sugiere realizar una revisión 48 a 72 horas posterior a la cirugía. Se recomienda además un control endoscópico semanal hasta superar la neutropenia, y, posteriormente, de manera mensual por 6 meses $^{11}$. Se ha reportado que los pacientes que sobreviven a RSFIA tienen un riesgo significativo de complicaciones tardías como mucoceles en cavidades paranasales, por lo que algunos autores sugieren realizar además imágenes sinusales a los 6-12 meses ${ }^{24}$.

\section{Pronóstico}

El pronóstico de los pacientes que desarrollan RSFIA es grave si no se diagnostica a tiempo, cuya mortalidad rara vez es inferior al $50 \%{ }^{23}$. En Chile existe solo un trabajo publicado en los últimos 15 años que hace referencia a la RSFIA, consistente en la casuística de 12 años de un hospital público terciario, que constató que la mortalidad de 14 casos de mucormicosis rino-órbito-craneal fue de $50 \% 25$.

De acuerdo a la revisión sistemática de Turner y cols, se pueden distinguir factores pronósticos positivos y negativos estadísticamente significativos mediante análisis multivariado. Por un lado, los factores pronósticos positivos corresponden a la resección quirúrgica abierta o endoscópica y la diabetes mellitus, y por otro, los factores pronósticos negativos corresponden a la invasión intracraneana y la edad avanzada ${ }^{3}$. Otros estudios retrospectivos han reportado mejor sobrevida en pacientes sometidos a resecciones quirúrgicas completas ${ }^{26}$, con un peor resultado relacionado a la 
extensión de la RSFIA y la enfermedad subyacente, sin existir diferencias de acuerdo al microorganismo aislado ${ }^{1,8}$. Se ha visto además que elementos clínicos como la tumefacción facial, el compromiso del septum nasal y la menor duración de los síntomas, así como elementos de laboratorio como la neutropenia severa y la elevación de la proteína C reactiva (PCR) plasmática, se relacionan con peor pronóstico ${ }^{27}$. Elevaciones de PCR plasmática superiores a $55 \mathrm{mg} / \mathrm{L}$ constituirían un factor pronóstico independiente, por lo que se sugiere su apropiado monitore $0^{27}$.

Otro estudio evaluó la mortalidad en relación a los subsitios afectados. De los 10 subsitios evaluados (senos frontal, maxilar, esfenoidal y etmoidal, tabique nasal, piso nasal y paladar duro, órbita, base de cráneo, pared nasal lateral e intracraneal), se encontró que el compromiso del tabique nasal se asociaba significativamente a mayor mortalidad. Se encontró además una diferencia significativa en la mortalidad de los casos de infección en 3 o más subsitios respecto a menos de 3 , siendo de $100 \%$ y $33,33 \%$, respectivamente ${ }^{6}$.

\section{Rinosinusitis fúngica invasiva en población pediátrica}

Con el advenimiento de terapias más eficaces en patologías pediátricas, se ha constatado un aumento de pacientes inmunosuprimidos y con ello la incidencia de infecciones oportunistas como la RSFIA ${ }^{28}$. Al igual que en el adulto, debe existir un alto índice de sospecha para la detección precoz de la RSFIA en población pediátrica de riesgo. De acuerdo a un trabajo retrospectivo reciente, el síntoma más frecuentemente reportado fue la fiebre (93\%), seguido de dolor facial (64\%) y síntomas sinusales inespecíficos $(58 \%)$. Con menor frecuencia se reportaron otros síntomas como rinorrea (42\%), cambios visuales $(29 \%)$ y tumefacción facial o palatina $(8 \%)^{29}$.

Una revisión sistemática de rinosinusitis fúngica invasiva en población pediátrica, llevada a cabo por Smith y cols, evidenció que la enfermedad subyacente más frecuente es la leucemia linfocítica aguda (LLA) en $44,6 \%$ de los casos, seguida de la leucemia mielocítica aguda (LMA), con $30,1 \%{ }^{28}$. El Aspergillus fue el microorganismo más frecuente- mente aislado en pacientes pediátricos (47\% de los casos), seguido por Mucor (8\%), Alternaria (8\%), Candida (4\%), Fusarium (4\%) y Rhizopus $(2 \%)$, con $27 \%$ restante desconocido o no especificado ${ }^{28}$. El RAN menor a 600 cél $/ \mathrm{mL}$ se presentó en el $99 \%$ de los casos, con una duración promedio de neutropenia de 2 semanas. La mortalidad global fue de $46 \%$, reconociendo mediante un análisis univariado al dolor facial como único predictor negativo de mortalidad, probablemente explicado porque ocurre precozmente en el curso de la RS$\mathrm{FIA}^{28}$. En otro estudio reciente, el único parámetro relacionado significativamente con la sobrevida fue el RAN promedio ${ }^{29}$. Al igual que en el adulto, el tratamiento antifúngico, junto a la intervención quirúrgica temprana y agresiva, son parte esencial del manejo de la RSFIA ${ }^{29}$. En la literatura chilena de los últimos 15 años, se describen 2 casos de pacientes pediátricos diagnosticados de mucormicosis rinoórbito-craneal aguda, uno con diabetes mellitus en contexto de cetoacidosis y otro con LLA, ambos con buena respuesta al tratamiento propuesto ${ }^{25}$.

\section{CONCLUSIONES}

La rinosinusitis fúngica invasiva sigue teniendo una alta tasa de mortalidad. Los factores predisponentes más comunes son la diabetes mellitus descompensada en adultos y las neoplasias malignas hematológicas en los niños. El nivel de sospecha clínica de la RSFIA debe mantenerse alto, a pesar de un resultado negativo en la biopsia, sobre todo en pacientes con neutropenia importante, fiebre persistente $y / 0$ signos locales nasosinusales, en la presencia de enfermedad unilateral, evidenciado endoscópica y/o imagenológicamente. El análisis de muestras de sección congelada es una herramienta confiable, que puede guiar el debridamiento endoscópico, y que ayudaría a evitar un procedimiento extenso que cause mayor morbilidad iatrogénica. En el manejo de estos pacientes es fundamental la creación de un equipo tratante multidisciplinario, constituido de otorrinolaringólogos, oncólogos, infectólogos, neurólogos, internistas, entre otros profesionales, para buscar la corrección de la enfermedad aguda y las condiciones subyacentes. 


\section{BIBLIOGRAFÍA}

1. Ergun 0, Tahir E, Kuscu 0, Ozgen B, Yilmaz T. Acute Invasive Fungal Rhinosinusitis: Presentation of 19 Cases, Review of the Literature, and a New Classification System. J Oral Maxillofac Surg 2017; 75: 767.e1-e9.

2. de Shazo RD, O'Brien M, Chapin K, Soto-Aguilar M, Gardner L, Swain R. A new classification and diagnostic criteria for invasive fungal sinusitis. Arch Otolaryngol Head Neck Surg 1997; 123 : 1181-8.

3. Turner JH, Soudry E, Nayak JV, Hwang PH. Survival outcomes in acute invasive fungal sinusitis: a systematic review and quantitative synthesis of published evidence. Laryngoscope 2013; 123: 1112-8.

4. Finkelstein A, Contreras D, Pardo J, Cruz JP, Gonzalez C, Constanza Beltrán M, Fonseca X. Paranasal sinuses computed tomography in the initial evaluation of patients with suspected invasive fungal rhinosinusitis. Eur Arch Otorhinolaryngol 2011; 268: 1157-62.

5. Gillespie MB, Huchton DM, O'Malley BW. Role of middle turbinate biopsy in the diagnosis of fulminant invasive fungal rhinosinusitis. Laryngoscope 2000; 110: 1832-6.

6. Foshee J, Luminais C, CAsey J, et al. An evaluation of invasive fungal sinusitis outcomes with subsite analysis and use of frozen section analysis. Int Forum Allergy Rhinol 2016; 6: 807-11.

7. Kasapoglu F, Coskun H, Ozmen OA, Akalin H, Ener B. Acute invasive fungal rhinosinusitis: evaluation of 26 patients treated with endonasal or open surgical procedures. Otolaryngol Head Neck Surg 2010; 143: 614-20.

8. Valera FC, do Lago T, Tamashiro E, Yassuda CC, Silveira F, Anselmo-Lima WT. Prognosis of acute invasive fungal rhinosinusitis related to underlying disease. Int J Infect Dis 2011; 15: e841-4.

9. Melancon CC, Clinger JD. The Use of Frozen Section in the Early Diagnosis of Acute Invasive Fungal Sinusitis. Otolaryngol Head Neck Surg 2017; 157: 314-9.

10. DelGaudio JM, Clemson LA. An early detection protocol for invasive fungal sinusitis in neutropenic patients successfully reduces extent of disease at presentation and long term morbidity. Laryngoscope 2009; 119: 180-3.
11. Epstein VA, Kern RC. Invasive fungal sinusitis and complications of rhinosinusitis. Otolaryngol Clin North Am 2008; 41: 497-524.

12. Ni Mhurchu E, Ospina K, Janjua AS, Shewchuk JR, VERTINSKY AT. Fungal Rhinosinusitis: A Radiological Review With Intraoperative Correlation. Can Assoc Radiol J 2017; 68: 178-86.

13. Middlebrooks EH, Frost CJ, De Jesus R0, Massini TC, Schmalfuss IM, Mancusso AA. Acute Invasive Fungal Rhinosinusitis: A Comprehensive Update of CT Findings and Design of an Effective Diagnostic Imaging Model. AJNR Am J Neuroradiol 2015; 36: 1529-35.

14. Slonimsky G, Slonimsky E, Yakirevitch A, et al. The significance of Computed Tomography in invasive paranasal mucormycosis. Rhinology 2018; 56: 54-8.

15. Groppo ER, El-Sayed IH, Aiken AH, Glastonbury CM. Computed tomography and magnetic resonance imaging characteristics of acute invasive fungal sinusitis. Arch Otolaryngol Head Neck Surg 2011; 137: 1005-10.

16. Aribandi M, McCoy VA, Bazan C 3rd. Imaging features of invasive and noninvasive fungal sinusitis: a review. Radiographics 2007; 27: 1283-96.

17. С Hо YR, KIm JH, Mın HS, et AL. Acute invasive fungal rhinosinusitis: MR imaging features and their impact on prognosis. Neuroradiology 2018; 60: 715-23.

18. Montone KT. Pathology of Fungal Rhinosinusitis: A Review. Head Neck Pathol 2016; 10: 40-6.

19. Hennessy M, McGinn J, White B, Payne S, Warrick JI, Crist H. Frozen Section as a Rapid and Accurate Method for Diagnosing Acute Invasive Fungal Rhinosinusitis. Otolaryngol Head Neck Surg 2018; 159: 576-80.

20. Papagiannopoulos P, Lin DM, Al-Khudari S, et AL. Utility of intraoperative frozen sections in surgical decision making for acute invasive fungal rhinosinusitis. Int Forum Allergy Rhinol 2017; 7: 502-7.

21. Cho HJ, Hong SD, Kim HY, Chung SK, Dhong HJ. Clinical implications of serum galactomannan measurement in patients with acute invasive fungal rhinosinusitis. Rhinology 2016; 54: 336-41.

22. Huang YD, Yu HW, XIA SW, Kang ZH, He YS, Han DY. Expression of maspin in invasive fungal rhinosinusitis. J Laryngol Otol2017; 131: 150-4. 
23. Deshazo RD. Syndromes of invasive fungal sinusitis. Med Mycol 2009; 47: S309-14.

24. Monroe MM, Mclean M, Sautter N, Wax MK, Andersen PE, Smith TL, Gross ND. Invasive fungal rhinosinusitis: a 15-year experience with 29 patients. Laryngoscope 2013; 123: 1583-7.

25. Rahal M, Moreno M, Villa J. Mucormicosis rinoorbito-cerebral: Experiencia en doce años. Rev Otorrinolaringol Cir Cabeza Cuello 2008; 68: 27-34.

26. Roxbury CR, Smith DF, Higgins TS, et al. Complete surgical resection and short-term survival in acute invasive fungal rhinosinusitis. Am J Rhinol Allergy 2017; 31: 109-16.
27. Cho HJ, Jang MS, Hong SD, Chung SK, Kim HY, Dhong HJ. Prognostic factors for survival in patients with acute invasive fungal rhinosinusitis. Am J Rhinol Allergy 2015; 29: 48-53.

28. Smith A, Thimmappa V, Shepherd B, Ray M, SHEYn A, THOMPson J. Invasive fungal sinusitis in the pediatric population: Systematic review with quantitative synthesis of the literature. Int J Pediatr Otorhinolaryngol 2016; 90: 231-5.

29. Green KK, Barham HP, Allen GC, Chan KH. Prognostic Factors in the Outcome of Invasive Fungal Sinusitis in a Pediatric Population. Pediatr Infect Dis J 2016; 35(4): 384-6. 